\title{
Study of the alkali treatment effect on the mechanical behavior of the composite unsaturated polyester-Alfa fibers
}

\author{
Azzedine Benyahia $^{1, a}$, Abdellah Merrouche ${ }^{1}$, Zine El Abidine Rahmouni ${ }^{2}$, \\ Mansour Rokbi ${ }^{3}$, Walter Serge ${ }^{4}$ and Zinat Kouadri ${ }^{1}$ \\ 1 Laboratoire des Matériaux Inorganiques, Université de M'sila, 166 rue Echbilia, 28000 M'sila, Algerie \\ 2 Laboratoire de développement des géomatériaux, Université de M'sila, 166 rue Echbilia, 28000 M'sila, Algerie \\ 3 Laboratoire des Matériaux Non Métalliques, UFAS Sétif 19000, Algerie \\ 4 École nationale supérieure de chimie de Mulhouse, France
}

Received 22 June 2013, Accepted 26 November 2013

\begin{abstract}
In this paper, composites based on unsaturated polyester resin reinforced with short Alfa fibers are studied in detail. Alfa fibers have been previously treated with various concentrations $\mathrm{NaOH}(1,3,5$, $7 \%$ ) during $24 \mathrm{~h}$. The influence of alkali treatment on fiber morphology is analyzed. Analysis by FTIR and X-ray diffraction showed physico-chemical changes in Alfa fiber treated surfaces. SEM observations also helped to highlight these changes. The results of static tests on composites showed improvements in tensile and flexural strengths of composites reinforced with the treated fibers, particularly the composite with treated fibers at $7 \% \mathrm{NaOH}$. These improvements were about $30 \%$ and $50 \%$, respectively, compared to untreated fiber reinforced composite.
\end{abstract}

Key words: Alkali treatment / mechanical properties / natural fibers / adhesion / crystallinity index

Résumé - Étude de l'effet du traitement alcalin de fibres naturelles sur le comportement mécanique du composite : polyester insaturé-fibre Alfa. Dans cet article, des composites à base de résine polyester insaturée renforcée par des fibres Alfa courtes sont étudiés. Les fibres Alfa ont été précédemment traitées avec différentes concentrations $\mathrm{NaOH}(1,3,5,7 \%)$ durant $24 \mathrm{~h}$. L'influence du traitement alcalin sur la morphologie des fibres a été analysée. Des analyses par FTIR et par la diffraction des rayons $\mathrm{X}$ ont montré des changements physico-chimiques dans les surfaces des fibres Alfa traitées. Des observations MEB ont permis aussi de mettre en évidence ces modifications. Les résultats des tests statiques sur les composites ont montré des améliorations de résistance en traction et en flexion des composites renforcés par des fibres traitées, en particulier le composite à fibres traitées à $7 \% \mathrm{NaOH}$. Ces améliorations sont d'environ $30 \%$ et $50 \%$, respectivement, comparées au composite à renfort non traité.

Mots clés : Traitement alkaline / propriétés mécaniques / fibres naturelles / adhésion / index de cristallinité

\section{Introduction}

Polymer composites are currently used for a wide range of applications. However, they do not easily undergo to biodegradation, resulting in generation of solid waste, which cause environmental pollution. To meet this challenge, researchers are focusing their attention on producing bio-degradable composites with natural fibers/fabrics which are termed as green composites. By using sisal, banana, bamboo, Alfa, coir, etc., several green composites have been already developed and their properties

${ }^{a}$ Corresponding author: azzedinebenyahia@yahoo.fr have been studied [1-4]. Natural fiber composites are not only biodegradable and renewable but also possess several other advantages such as light weight, low cost, high specific strength, high modulus, reduced tool wear and safe manufacturing process $[1,2]$. Several applications of natural fiber composites can be found in construction, packaging, furniture and auto-motive fields. Most of the interiors of the automobiles, like door panels, trunk liners, seal backs, packages, engine and transmission covers are made by using natural fiber composites. Despite several merits, they also possess few drawbacks such as poor wettability, incompatibility with some polymeric matrices and high 
Table 1. Different manufacturing composites.

\begin{tabular}{cccc}
\hline Composite & Designation & $\begin{array}{c}\text { Fibers loading } \\
\%(\mathrm{w} / \mathrm{w})\end{array}$ & $\begin{array}{c}\text { Fibers loading } \\
\%(\mathrm{~V} / \mathrm{V})\end{array}$ \\
\hline Untreated Alfa fibers/UP & T0000 & 10 & \\
1\% Alkali treated Alfa fibers/UP & T0124 & 9.8 & 40 \\
3\% Alkali treated Alfa fibers/UP & T0324 & 9.9 & \\
$5 \%$ Alkali treated Alfa fibers/UP & T0524 & 10.1 & \\
$7 \%$ Alkali treated Alfa fibers/UP & T0724 & 9.7 & \\
\hline
\end{tabular}

moisture absorption by the fibers [5]. To overcome this problem, specific physical and chemical treatments were suggested for surface modification of fibers by investigators. Alkali treatment is one of the simplest and most effective surface modification techniques which is widely used in natural fiber composites $[2,3]$.

In the present study, both untreated and alkali treated short fibers (after grinding) were used as reinforcement in unsaturated polyester resin composites. Tensile and flexural properties were determined at different concentrations of $\mathrm{NaOH}(0 \%, 1 \%, 3 \%, 5$ and 7 wt $\%)$ during $24 \mathrm{~h}$ at ambient temperature. The alkali treatment was found to be effective in improving the tensile and flexural properties of Alfa fiber reinforced polyester resin.

\section{Experimental}

\subsection{Fibers and resin}

Alfa (Stipa Tenacissima L.) fibers were used as reinforcement. These fibers were obtained from a semi-arid region (Boussâada - Algeria). Alfa grass is a tussock grass; it is constituted of stems with a cylindrical shape and a maximum height of about $1 \mathrm{~m}$. Once the Alfa fibers were harvested, they were washed with water $(2 \%$ detergent solution) to remove the contaminants and adhering dirt. Thereafter, fibers were cut into small lengths $(0.2$ to $0.4 \mathrm{~cm})$. The average diameter of used Alfa fibers was in the range of $90-500 \mu \mathrm{m}$. Similar to other natural fibers, the major constituents of Alfa fibers are cellular, hemicellulose and lignin. The chemical composition of Alfa grass is composed of $45 \%$ of cellulose, $25 \%$ pentozane, $23 \%$ lignin, $5 \%$ wax and $2 \%$ of ash [6]. In this investigation; commercial isophthalic polyester (UP) ISO for stratification was used as a resin.

\subsection{Alkali treatment}

Alfa fibers were soaked in 1, 3, 5, and 7 wt $\% \mathrm{NaOH}$ solutions at $25^{\circ} \mathrm{C}$ for $24 \mathrm{~h}$, maintaining a liquor ratio of 15:1. The fibers were washed for several times with water to remove any alkali solution sticking on their surface, neutralized with dilute acetic acid and then washed again with water. Finally, the resulted fibers were dried at $80{ }^{\circ} \mathrm{C}$ for $48 \mathrm{~h}$. The fibers were arranged in the mould $\left(300 \times 300 \times 5 \mathrm{~mm}^{3}\right)$; extreme care was taken to get uniform distribution. A mixture of diluent with $5 \mathrm{wt} \% \mathrm{UP}$ resin was sprayed onto the random mat so that it can handle during the molding [2]. Mould was then closed and a pressure was applied to get it as a single mat. Composites were made using a mould with the same dimensions previously cited. The resin was cured using $1 \%$ of methyl ethyl ketone peroxide catalyst and $0.5 \%$ of cobalt-naphthanate accelerator. The manufacturing of the structural composite consists in soaking two random mats with polyester resin in the mould impression, then to press them with the mould cover. Five plates of composites are manufactured (Table 1) using hand lay-up technique [7]. The filler content was fixed at $2 \mathrm{wt} \%$ for all the composite materials.

\subsection{Chemical and physical characterizations of the fibers}

In the present study, the infrared spectra of fibers before and after alkali treatment were measured with a FTIR (SHIMADZU, series 8300). Powdered fiber pelletized with potassium bromide was used for recording the spectra. Transmittance was measured over a range from 4000 to $400 \mathrm{~cm}^{-1}$. In order to assess the influence of the treatment on the fiber crystallinity, XRD analysis was applied using a X'Pert High Score PW3209 diffractometer. The equatorial diffraction patterns $(2 \theta)$ were recorded from 10 to $40^{\circ}$ using $\mathrm{Cu}-\mathrm{K} \alpha$ radiation at $40 \mathrm{KV}$ and $20 \mathrm{~mA}$. The Crystallinity index (CrI) were calculated according to the Segal empirical method:

$$
\operatorname{CrI}(\%)=100 \times\left[\left(I_{002}-I_{\mathrm{am}}\right) / I_{002}\right]
$$

where: $\mathrm{I}_{002}$ is the maximum intensity of the 002 lattice reflection of the cellulose crystallographic form (I) at $2 \theta=22^{\circ}$ and $\mathrm{I}_{\mathrm{am}}$ is the intensity of diffraction of the amorphous at $2 \theta=18^{\circ}$. The treated and untreated Alfa fibers morphology were observed by scanning electron microscopy (SEM) using a Cambridge S200 microscope. One set of samples from each type of fibers was examined.

\subsection{Mechanical tests}

Tensile and flexural tests of the composite specimens were carried out at a cross head speed of $1 \mathrm{~mm} . \mathrm{min}^{-1}$. For the flexural test, specimens were submitted to the three-point bending test. The load displacement curves were obtained. The flexural strength and Young's modulus of composites were calculated using the following relationships:

Flexural strength

$$
\sigma_{\mathrm{f}}=3 P L / 2 b d^{2}
$$




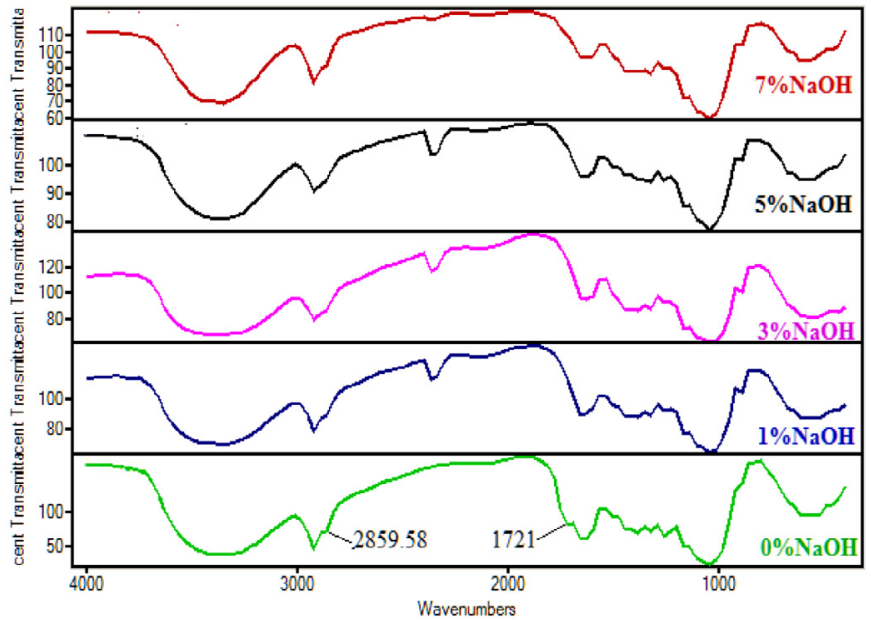

Fig. 1. FTIR Spectra of the treated and untreated Alfa fibers.

Flexural modulus:

$$
E_{\mathrm{f}}=L^{3} m / 4 b d^{3}
$$

where: $L(\mathrm{~mm})$ is the support span; $b$ the width of the specimen; $d$ the thickness; $P(\mathrm{~N})$ the maximum load; and $m$ the slope of the initial straight line portion of the loaddisplacement curve.

\section{Results and discussion}

\subsection{Effect of alkali treatment on mechanical properties}

In this study, the effects of alkali treatment $(1,3,5$ and $7 \% \mathrm{NaOH}$ ) on Alfa fiber properties were investigated. Changes regarding treated and untreated Alfa fibers morphology by FTIR measurement, SEM and XRD analysis were presented. The FTIR spectra of untreated and alkali treated Alfa fiber are presented in Figure 1. The broad absorption band observed in the $3359.8 \mathrm{~cm}^{-1}$ was related to the hydrogen bonding $(\mathrm{OH})$ stretching vibration. It appeared weaker in intensity in the spectrum after the $5 \%$ $\mathrm{NaOH}$ treatment. Vibration peak at $2916.2 \mathrm{~cm}^{-1}$, corresponding to the stretching of the $\mathrm{C}-\mathrm{H}$ aliphatic group was included in almost all natural fibers. This peak decreased due to the removal of hemicelluloses [8]. It is also clearly noticeable that the band around $1731.9 \mathrm{~cm}^{-1}$ corresponding to non-cellulosic components (pectin, lignin and hemicelluloses) was disappeared, suggesting their removal from the fiber when this one was treated by $5 \% \mathrm{NaOH}$ solution. The peak at $1654.8 \mathrm{~cm}^{-1}$ would be due to the presence of lignin [9]. The $1454 \mathrm{~cm}^{-1}$ band was attributed to the $\mathrm{CH}_{2}$ bending of cellulose. The peak at $1253.6 \mathrm{~cm}^{-1}$, which is $\mathrm{C}=\mathrm{O}$ stretch of acetyl group of lignin was reduced. This was due to the partial removal of lignin from the fiber surface. Alkali treatment is expected to reduce the hydrogen bonding in cellulose hydroxyl groups by the removal of the carboxyl group [1]. We noted a decreasing of the pick relative to $(\mathrm{OH})$ bound of cellulose; this was also the case
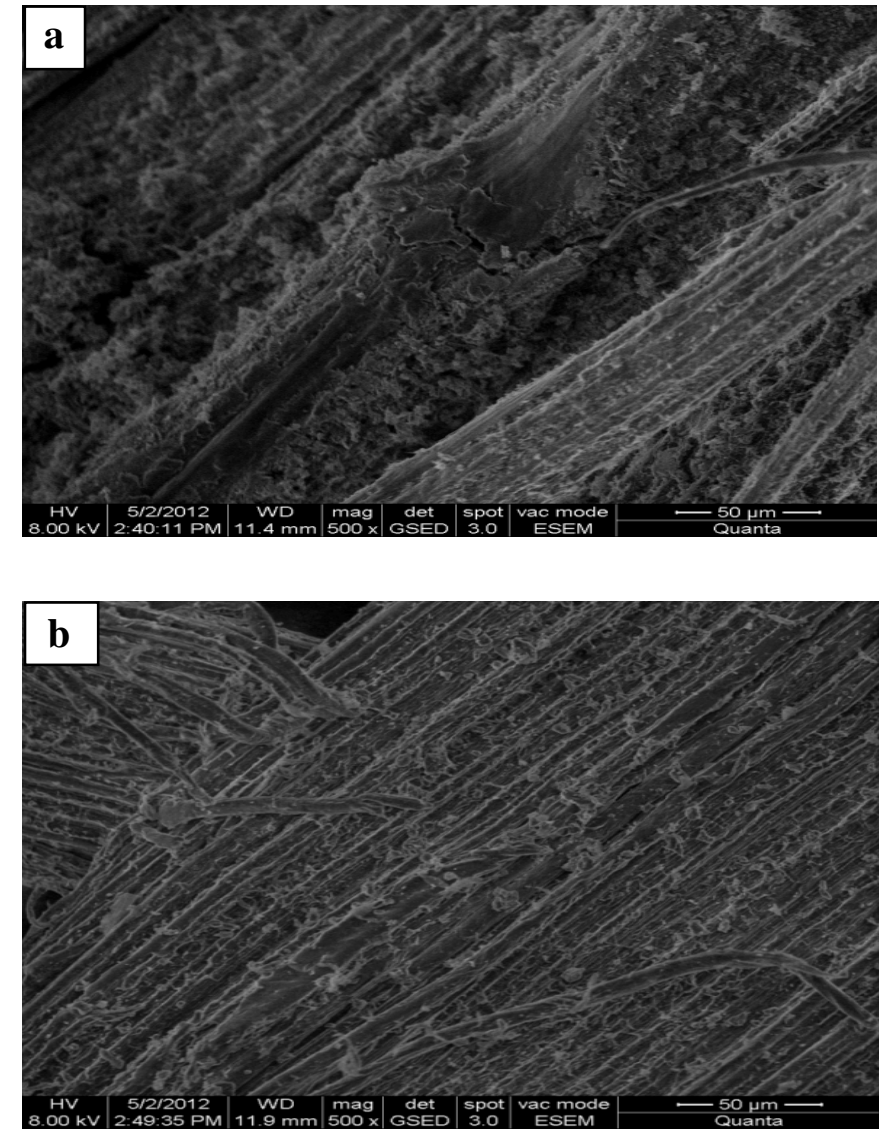

Fig. 2. SEM micrographs of: (a) untreated; and (b) treated Alfa fibers.

at the peak around $1033 \mathrm{~cm}^{-1}$. We concluded that the numbers of hydroxyl groups $(\mathrm{OH})$ were decreased. In addition, a part of lignin was removed from the fiber. Both consequences were related to the direct effect of an adequate Alfa fiber treatment by $\mathrm{NaOH}$ solution.

Figure 2 shows the surface morphology of raw and alkali treated fibers. The alkali treatment leads to significant differences in the fiber surface morphology [1]. As shown in Figure 2a, the surface of an untreated Alfa fiber was covered with a layer of substances, which may include pectin, lignin, and other impurities $[2,3]$. After $\mathrm{NaOH}$ treatment (Fig. 2b), it is seen that the treatment has removed the surface debris from the fiber [11], most of the lignin and pectin are removed resulting in a rougher surface. This rough surface facilitates both mechanical interlocking and bonding reaction due to the exposure of the hydroxyl groups to the matrix, thereby increasing the fiber-matrix adhesion [12]. It was found that the macro grass fibers are composed of smaller individual single fibers held together by material in the inter-fibrillar region [13]. As a consequence: (i) the interfacial interaction between the fiber and matrix is possible; (ii) this increases the effective available surface contact area with the matrix; and (iii) the possibility of load transfer between the matrix and the reinforcing fibers [2]. The XRD analyses of the fibers were carried out. Results are given 


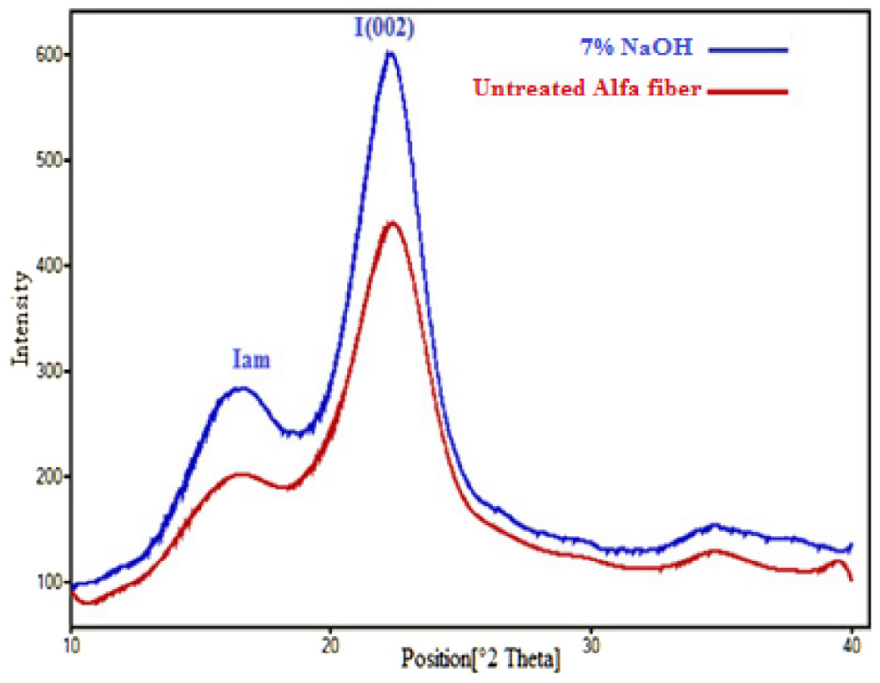

Fig. 3. X-Ray diffraction patterns of treated and untreated Alfa fibers.

Table 2. Crystallinity index of untreated and treated Alfa fibers.

\begin{tabular}{cccc}
\hline Composites & $I(002)$ & $I_{\mathrm{am}}$ & $\mathrm{CrI}(\%)$ \\
\hline Untreated Alfa fibers/UP & 274 & 155 & 43.43 \\
$7 \%$ Alkali treated Alfa fibers/UP & 462 & 127 & 51.52 \\
\hline
\end{tabular}

in Figure 3 and the crystallinity index (CrI) was calculated according to the Segal empirical method described in the experimental section as presented in Table 2, as seen from Figure 3,

Alfa fiber exhibited a typical cellulose I pattern, well defended peak at $2 \theta=22^{\circ}$. The reflections peak at $22^{\circ}$ corresponds to the 002 crystallographic plane of the cellulose I lattice. Maximum value of the intensity is clearly observed in the fiber treated with $7 \% \mathrm{NaOH}$. The CrI for the raw fiber increased of about $16 \%$. This increasing indicated the improvement in the cellulose structure, and finally contributed to enhancing the tensile strength of the fiber.

\subsection{Effect of alkali treatment on mechanical properties}

The effect of surface treatment on the mechanical properties of tested materials can be readily assessed from Table 3. The material T0724 displayed the highest tensile strength (25.11 MPa) compared to the untreated Alfa fibers composites (17.48 MPa). This may be due to the bonding of the fiber with the polyester matrix thereby improving the fiber-matrix interaction [2]. The significance of alkali treatment is the disruption of hydrogen bonding in the fiber surface, thus increasing surface roughness [12]. The alkali treatment improved fiber matrix interaction by the removal of lignin and hemicellulose, which led to the better incorporation of fiber with the matrix [14].
Table 3. Tensile and flexural properties of tested materials.

\begin{tabular}{cccccc}
\hline & \multicolumn{5}{c}{ Materials } \\
\hline Properties & T0000 & T0124 & T0324 & T0524 & T0724 \\
\hline Tensile strength (MPa) & 17.48 & 18.09 & 23.42 & 24.33 & 25.11 \\
Tensile modulus (GPa) & 0.414 & 0.534 & 0.889 & 0.990 & 0.960 \\
Flexural strength (MPa) & 16.65 & 22.62 & 25.51 & 26.83 & 3312 \\
Flexural modulus (GPa) & 1.05 & 1.57 & 1.63 & 1.75 & 1.89 \\
\hline
\end{tabular}

The results have shown that the tensile strength and tensile modulus increased with the increasing of $\mathrm{NaOH}$ concentration. The alkali treatments of Alfa fibers have also a significant effect on flexural properties. From Table 3 , it was clearly seen that the material T0724 showed the highest improvement in terms of flexural strength; which increased by 50\% compared to T0000 material. This addition remarkable in flexural strength may be an indication of better adhesion between the polyester and modified Alfa fibers [2]. It seemed that we need to widen the margin of the $\mathrm{NaOH}$ concentration to determine the desired optimum.

\section{Conclusions}

In this study, Alfa fibers were treated with 1, 3, 5 and $7 \%$ sodium hydroxide solution for $24 \mathrm{~h}$. The tensile and flexural properties of their composites were increased after alkali treatment and became better for $7 \%$ alkali treatment. Compared to the untreated fibers composites, the increase in tensile and flexural strengths of $7 \%$ treated composite were respectively of about $30 \%$ and $50 \%$; which were due to high enhancement in the interfacial adhesion of the fiber with the polyester resin. The SEM analyses revealed that $\mathrm{NaOH}$ treatment modified the fiber morphology. Alfa fiber can be obtained at a relatively low cost compared to glass fiber reinforcements, and then these fibers have a very promising future in composite material.

\section{References}

[1] B.F. Yousif, A. Shalwan, C.W. Chin, K.C. Ming, Flexural properties of treated and untreated kenaf/ epoxy composites, Mater. Design 40 (2012) 378-385

[2] M. Rokbi, H. Osmani, A. Imad, N. Benseddiq, Effect of Chemical treatment on Flexure Properties of Natural Fiber-reinforced Polyester Composite, Procedia Engineering 10 (2011) 2092-2097

[3] Y. Cao, S. Shibata, I. Fukumoto, Mechanical properties of biodegradable composites reinforced with bagasse fibre before and after alkali treatments, Composites: Part A 37 (2006) 423-429

[4] T. Alsaeed, B.F. Yousif, H. Ku, The potential of using date palm fibres as reinforcement for polymeric composites, Mater. Design 43 (2013) 177-184 
[5] D. Bachtiar, S.M. Sapuan, M.M. Hamdan, The effect of alkaline treatment on tensile properties of sugar palm fibre reinforced epoxy composites, Mater. Design 29 (2008) $1285-1290$

[6] M.C. Paiva, I. Ammar, A.R. Campos, R. Ben Cheikh, A.M. Cunha, Alfa fibres: Mechanical, morphological and interfacial characterization, Compos. Sci. Technol. 67 (2007) 1132-1138

[7] S. Ben Brahim, R. Ben Cheikh, Influence of fibre orientation and volume fraction on the tensile properties of unidirectional Alfa-polyester composite, Compos. Sci. Technol. 67 (2007) 140-147

[8] M.S. Sreekala, S. Thomas, Effect of fiber surface modification on water-sorption characteristics of oil palm fibers, Compos. Sci. Technol. 63 (2003) 861-869

[9] A. Bessadok, S. Marais, F. Gouanvé, L. Colasse, I. Zimmerlin, S. Roudesli, M. Métayer, Effect of chemical treatments of Alfa (Stipa tenacissima) fibres on watersorption properties, Compos. Sci. Technol. 67 (2007) $685-697$
[10] L. Boopathi, P.S. Sampath, K. Mylsamy, Investigation of physical, chemical and mechanical properties of raw and alkali treated Borassus fruit fiber, Composites Part B 43 (2012) 3044-3052

[11] C. Gonzalez-Murillo, M.P. Ansell, Mechanical properties of henequen fibre/epoxy resin composites, Mech. Compos. Mater. 45 (2009) 435-442

[12] L. Yan, N. Chouw, X. Yuan, Improving the mechanical properties of natural fiber fabric reinforced epoxy composites by alkali treatment, J. Reinforced Plastics and Composites 31 (2012) 425-437

[13] A.K. Bledzki, J. Gassan, Composites reinforced with cellulose based fibres, Progress in Polymer Science 24 (1999) $221-274$

[14] I. Nor Azowa, H. Kamarul Arifin, A. Khalina, Effect of Fiber Treatment on Mechanical Properties of Kenaf Fiber-Ecoflex Composites, J. Reinforced Plastics and Composites 29 (2010) 2192-2197 